\title{
Describing Creativity with Card Sorting and Processing.
}

\author{
Shaun McWhinnie \\ University of Dundee \\ School of Computing \\ s.mcwhinnie@dundee.ac.uk
}

\author{
Shaleph O'Neill \\ University of Dundee \\ School of Computing \\ s.j.oneill@dundee.ac.uk
}

\begin{abstract}
The paper describes our work in modelling creativity, in the hope of understanding the differences between those who use interactive technologies as party of their creative process and those who use traditional media.
\end{abstract}

Creativity, Methodologies

\section{INTRODUCTION}

This paper reports work from the EPSRC funded Making Sense of Creative Interactions project (\#EP/F053029/1). The goal of our work is to understand how creative individuals use and make sense of interactive software.

The exact properties of creativity often seem nebulous, however, this may be due to creativity being a presence of different types of creativity, some 'domain general' (i.e. present in all people engaged in creative work), and some 'specific' to the creative domain an individual works in. Sternberg (2005) summarizes various approaches to these multiple creativities. Our previous studies have utilized a method of enquiry called Repertory Grid Technique (RGT) (Kelly, 1963), to analyze people's description of their own creative practice. Kelly's method relies upon triadic elicitation methods. For example, consider 3 elements, A, B, and $C$. If we say $A$ and $B$ are similar, but different from $C$, and articulate why this is the case, we are describing one axis of value, or construct, within a participant's conceptualization of a particular domain. For the purposes of our work we are interested in what constructs people use in terms of their creative process, and where the similarities and differences are between people working in different fields. The first stage of our research involved doing individual studies, which revealed rich and nuanced descriptions of personal creative practice (McWhinnie, O'Neill, 2009). However, the individual nature of these studies made it impossible to make accurate comparisons between participants. A follow up study attempted to resolve this issue by structuring the RGT method into a 'focus-group' approach, where the elements of the study were determined by the group at the start of the session (McWhinnie, O'Neill, Valentine, 2009). This allowed us to compare the results between all the individuals within that particular group based study.

However, in moving beyond this study, we found ourselves in a classic 'Catch-22' situation. In order to compare between participants, over a range of studies it is necessary to have a stable set of 'universal base elements' which can be used across any group; in order to establish these 'base elements', it is necessary to have a rich understanding of the way in which a wide range of 'elements' are used by creative practitioners to describe their creative processes. Which one comes first? Our solution has been to utilise the wide range of elements gathered in our early RGT studies, and employ them in a card sorting exercise, aimed at understanding how a range of elements were consistently grouped together by all participants, essentially identifying some usable, generally applicable 'base elements' (relationships between words and phrases) necessary to describe creative practice.

\section{CARD SORTING}

Initially we collated all the elements from our early studies eliminating duplicates and those that were likely to lead to confusion (i.e. words that were too obtuse or esoteric). The remaining 103 elements were printed on laminated cards. We then asked 7 pairs of Interaction Design students to sort these 103 elements into 7 unnamed groups. We utilised pairs of students in order to ensure some consensus of opinion. As we are more interested in the constructs elicited, it could be argued that the 
choice of 7 elements is somewhat arbitrary, however, 7 elements gives a balance between variety and originality for the purposes of comparison (Fransella and Bannister, 1999). The sorted groups of elements were then analysed by a custom Processing script that looked for levels of correspondence of element placement across all the studies. For instance, where element ' $A$ ' appeared alongside element ' $B$ ' in all 7 card sort groups, we could surmise that they are $100 \%$ connected (within our sample), whereas where element ' $A$ ' appears alongside element ' $C$ ' in 6 of 7 groups, we can surmise they are $\sim 85 \%$ connected.

The results of these comparisons can be used in two main ways. Firstly, by completing further studies with different groups of practitioners from different fields, we will be able to identify the most strongly correlated elements, which provides a rigorous representation of the 7 most general element groupings that can be used for further RGT studies. This helps us to understand where the 'domain general' aspects of creativity lie. In addition to this, the creation of the processing software allows us to create dynamic visual representations of the data gathered. An advantage of using processing is that it is easily deployable via the web, allowing access to hundreds or possibly thousands of participants from many different fields. This is also a way of identifying which properties of the creative process are more 'domain specific'. For instance, by comparing the results of the media design students here with the results from a group of students engaged in a more traditional medium, such as drawing and painting, we may see differences across the groupings, indicating a fundamental difference in the way that the groups think. Additionally, this information can be extended to explore the similarities and differences between almost any groups of practitioner, for instance, the difference between artists and designers, between nationalities, between young and old etc. Our hope is that in developing this as a resource it may become a hub for discussion and a useful tool for researchers within the field of creativity study.

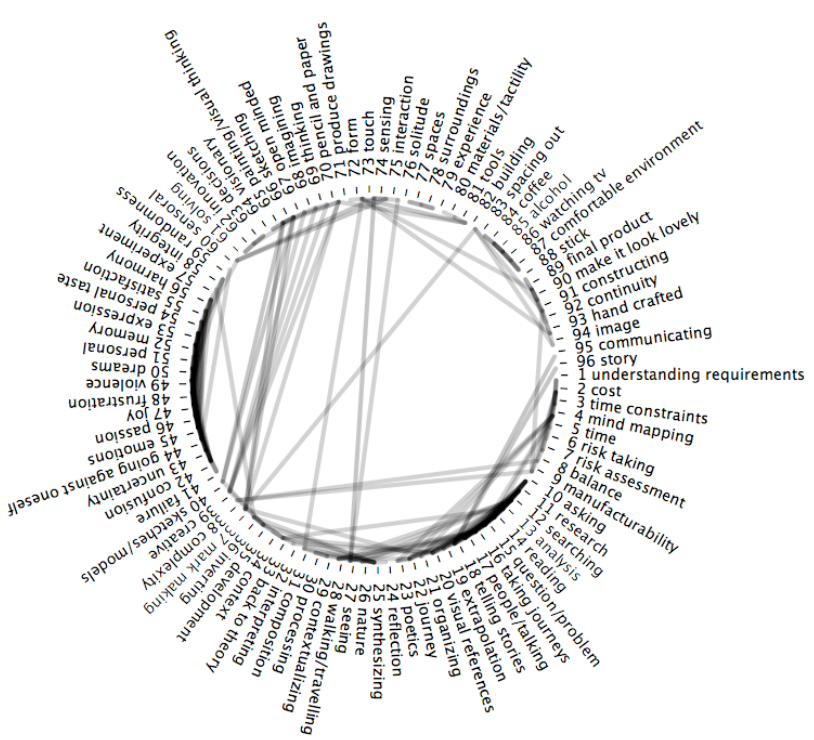

Figure 1. An example of processing output, showing links between terms.

\section{REFERENCES}

Sternberg R.J. (2005), Creativity or Creativities?, Int. J. Human-Computer Studies 63 370-382

Kelly, G. A. (1963) A Theory of Personality: the psychology of personal constructs. Norton, New York.

McWhinnie, S., O'Neill, SJ. (2009) Making sense of Creative Interactions Through Repertory Grid Technique. Create 2009, London, $1^{\text {st }}$ July 2009.

McWhinnie, S. O'Neill, SJ. Valentine, L. (2009) Random Thinking, Ordered Doing: Understanding group creative practice through repertory grid technique. Creativity and Cognition 2009, Berkeley, CA, $26^{\text {th }}$ October 2009.

Fransella, F., and Bannister, D. (1999) A Manual for Repertory Grid Technique. London, Academic Press, 